\title{
Lean manufacturing: 5 s y TPM, herramientas de mejora de la calidad. Caso empresa metalmecánica en Cartagena, Colombia*
}

Lean manufacturing: $5 \mathrm{~s}$ and TPM, quality improvement tools. Metalmechanical company case in Cartagena, Colombia

\section{Lean manufacturing: 5 s e TPM, ferramentas de melhoria da qualidade. Caso empresarial metalmecânica em Cartagena, Colombia}

Martha Sofía Carrillo Landazábal"

Fundación Universitaria Tecnológico Comfenalco, Colombia

Carmen Giarma Alvis Ruiz:*

Fundación Universitaria Tecnológico Comfenalco, Colombia

Yaniris Yaneth Mendoะa Álvarez ${ }^{* * *}$

Fundación Universitaria Tecnológico Comfenalco, Colombia

Harold Enrique Cohen Padilla****

Fundación Universitaria Tecnológico Comfenalco, Colombia

Citar como: Carrillo Landazábal, M. S., Alvis Ruiz, C. G., Mendoza Álvarez, Y. Y., y Cohen Padilla, H. E. (2019). Lean manufacturing: 5 s y TPM, herramientas de mejora de la calidad. Caso empresa metalmecánica en Cartagena, Colombia. SIGNOS - Investigación en sistemas de gestión, 11(1), 71-86. DOI: https://doi.org/10.15332/ s2145-1389.2019.0001.04

* Artículo de resultado de investigación.

** Estudiante doctoral en Ciencias Técnicas (Universidad Central de las Villas, Cuba), magíster en Administración, especialista en Administración Financiera, ingeniera industrial. Grupos de investigación: GIPIPQ y CIPTEP. Fundación Universitaria Tecnológico Comfenalco, Programa de Ingeniería Industrial, Cartagena, Colombia. Correo electrónico: invest.industrial@tecnologicocomfenalco.edu.co. ORCID: https:/orcid. org/0000-0002-5446-9010

** Magíster en Administración, ingeniera industrial. Grupos de investigación: CIPTEC. Fundación Universitaria Tecnológico Comfenalco, Programa de Ingeniería Industrial, Cartagena, Colombia. Correo electrónico: calvis@tecnologicocomfenalco.edu.co. ORCID: https://orcid. org/0000-0003-1128-0426

**** Magíster en Calidad, ingeniera industrial. Grupo de investigación: CIPTEC. Fundación Universitaria Tecnológico Comfenalco, Programa de Ingeniería Industrial, Cartagena, Colombia. Correo electrónico: ymendozaa@tecnologicocomfenalco.edu.co. ORCID: https://orcid. org/0000-0002-2694-7791

***** Magíster en Confiabilidad, ingeniero industrial. Grupo de investigación: CIPTEC. Fundación Universitaria Tecnológico Comfenalco, Programa de Ingeniería Industrial, Cartagena, Colombia. Correo electrónico: hcohen@tecnologicocomfenalco.edu.co. ORCID: https://orcid.org/000-00026394-790X. DOI: https://doi.org/10.15332/s2145-1389-4934 


\section{RESUMEN}

Todo proceso de mejora continua debe promover que las condiciones de trabajo se presenten de manera que los retrasos, las pérdidas de operatividad o cualquier fallo se reduzcan paulatinamente, de modo que el aprovechamiento de los recursos esté lo más cercano a lo óptimo posible. Es por ello que se ha trazado como objetivo primordial en este estudio establecer una propuesta de implementación de lean manufacturing por medio del uso de herramientas de producción esbelta y de confiabilidad de procesos, de modo que se promueva la búsqueda del beneficio mutuo en el aprovechamiento de oportunidades de mejora y optimización de recursos y procesos. Para tal hecho, se hicieron mediciones del sistema actual versus el propuesto 0 mejorado de manera que se facilitara la operación, el manejo de las rutinas de trabajo, el ambiente físico de las instalaciones y se elevara la motivación de todos en la empresa. Este tipo de investigación se presenta de manera descriptiva diseñado con base a las herramientas lean manufacturing como las cinco eses $(5 \mathrm{~s})$ y herramientas de mantenimiento en confiabilidad a través de sus conceptos y del uso del software Weibull ++6.

Palabras clave: lean manufacturing, mantenimiento productivo total, cinco eses (5 s), mejora continua.

\section{ABSTRACT}

Any process of continuous improvement must promote that working conditions are presented in such a way that delays, operational losses or any failure are gradually reduced, so that the use of resources is as close as possible to the optimum. For this reason, it has been established as the primary objective in this study to determine a lean implementation proposal, through the use of lean production and process reliability tools, so as to promote the pursuit of mutual benefit in the use of opportunities for resources and processes improvement and optimization. Thus, measurements of the current system were made versus the proposed or improved one, in order to facilitate the operation, the work routines management, the facilities physical environment and the motivation growth of the whole staff in the company. This type of research is presented in a descriptive way, based on the Lean Manufacturing tools such as the five "S"s (5 s) and reliability maintenance tools through their concepts and the use of Weibull ++ 6 software.

Keywords: Lean manufacturing, total productive maintenance, $5 \mathrm{~s}$, improvement continues.

\section{RESUMO}

Todo processo de melhora continua deve promover que as condições de trabalho se apresentem de maneira que os atrasos, as perdas de operabilidade ou qualquer falha se reduzam paulatinamente, de modo que 0 aproveitamento dos recursos seja o mais próximo do ótimo possível. É por isso que se tem traçado como objetivo primordial em este estudo estabelecer uma proposta de implementação do lean manufacturing por meio do uso de ferramentas de produção esbelta e confiabilidade de processos, de modo que se promova a procura do beneficio mutuo no aproveitamento de oportunidades de melhora e otimização dos recursos e processos. Para isso, fizeram-se medições do sistema atual versus o proposto ou melhorado de maneira que se facilitara a operação, o manejo das rotinas de trabalho, 0 ambiente físico das instalações e se elevasse a motivação de todos na empresa. Este tipo de pesquisa apresenta-se de maneira descritiva desenhada com base nas ferramentas lean manufacturing como os cinco esses (5 s) e ferramentas de manutenção em confiabilidade através de seus conceitos e do uso do software Weibull ++6. 
Palavras-chave: lean manufacturing, manutenção produtiva total, cinco esses $(5 \mathrm{~s})$, melhora continua.

\section{INTRODUCCIÓN}

En la gran mayoría de los países del hemisferio occidental, especialmente los subdesarrollados o en vía de desarrollo como Colombia, se ha evidenciado la ausencia de modelos científicos, técnicas y estrategias de trabajo realmente aplicadas a las pequeñas y medianas empresas, esto debido al poco apoyo financiero en investigación y desarrollo (I + D). Por ejemplo, en opinión de Jorge Núñez Jover, director de Posgrado de la Universidad de La Habana, si se considera el gasto interno bruto en investigación y desarrollo (GIBID) de Estados Unidos (167.01 miles de millones de dólares), la Unión Europea (117.67) y Japón (68.31), ese gasto es superior al $80 \%$ del total mundial $(428,58)$. Los más próximos son China (22.24) y los nuevos países industrializados (10.73); al mismo tiempo se habla que mientras el promedio mundial del GIBID en relación con el producto interno bruto (PIB) es de $1.8 \%$, la Unión Europea alcanza el 1.9, Estados Unidos el 2.8 y Japón el 2.8, Colombia sigue invirtiendo en I+D lo mismo que hace 21 años: $0.2 \%$ del PIB (Barrientos Marín, 2016) cuyo valor en 2016 era de $\$ 12.071$ millones de dólares, según informa la Universidad de los Andes de acuerdo a reportes de la agencia de inversiones Invest In Cartagena, 2017. Esto se debe principalmente a que dichas empresas tienen su génesis en el aprovechamiento de algunas oportunidades puntuales de trabajo que desembocaron en una incipiente estructura organizacional, carente en la mayor parte de las ocasiones de una planeación que lleve a la organización al aprovechamiento de los espacios, los recursos e incluso los impactos al medio ambiente. Colombia y en especial Cartagena no han sido ajenas a esta generalizada problemática que se evidencia con mayor preocupación en el sector metalmecánico, en donde se suma la presencia de personal laboralmente activo en condiciones muy bajas de capacitación formal o preparación técnica. Por tal razón, se hace necesario adaptar e implementar una filosofía desarrollada alrededor de los años cincuenta por la Toyota en Japón, denominada lean manufacturing, que conlleve al mejor desarrollo de los procesos, el ahorro de recursos y la satisfacción final de los usuarios en mejores condiciones de operatividad empresarial.

La propuesta de lean mamufacturing o fabricación esbelta en español, busca la manera de optimizar el sistema de producción, intentando eliminar o reducir todas las tareas que no añadan valor dentro del proceso de producción, convirtiéndose es una filosofía productiva que, al considerar valorar y controlar el despilfarro o desperdicio, centra su atención en la optimización de los recursos de la empresa. Según Stone (2012) y Holweg (2007), el desperdicio es todo aquello que no agrega valor al producto desde la perspectiva del cliente, y de igual forma comprende un conjunto de prácticas productivas para alcanzar dicho objetivo.

La adaptación de las herramientas del lean manufacturing pretende responder a la necesidad que tiene una empresa del sector metalmecánico automotriz de la ciudad de Cartagena, para identificar mejores prácticas en la ejecución de los procesos, comprometer a los trabajadores en los procesos de mejora, y que además de la satisfacción del cliente, estas prácticas y compromisos lleven al cumplimiento de los estándares de calidad requeridos. Las empresas muchas veces no consideran factores de influencia interna como son: la limpieza, el orden, el aprovechamiento de los espacios y de los recursos, así como el deterioro de los activos (máquinas, equipos, e instalaciones) por falta de buenas prácticas, llegando en algunos casos a presentarse incrementos en los costos por encima de los previstos para operación y entrega de productos o servicios por no realizar esos análisis. 
Uno de los fundamentos del lean es el funcionamiento al $100 \%$ de los equipos (Cuatrescasas y Torrel, 2010), indispensable para la implementación de este sistema productivo. Si no existe un sistema de mantenimiento, se podrían presentar fallas en los activos críiticos de la organización lo que afectaría negativamente la competitividad y rentabilidad de las organizaciones, que podría llevar incluso al cese de sus actividades. Es por esto la especial importancia del mantenimiento industrial que lo coloca en la posición de ser considerado como una unidad de negocio en la organización y ser tratado como tal.

\section{REVISIÓN DE LITERATURA}

\section{Lean manufacturing}

Lean manufacturing es una filosofía originaria de Japón, la cual tiene su génesis en el sistema de fabricación de Toyota, aunque se dice que dicha filosofía se inició, según Shah y Ward (2007), en el año 1927. Su contraparte americana, Ford, también planteó su propia filosofía de producción y principios y que en términos generales no distan una de la otra. Sea americana 0 japonesa, como efectivamente se reconoce, la filosofía lean se basa en la mejora continua de los procesos por lo que los siguientes sistemas de producción prometen allanar el camino en pro de la consecución de dichos resultados: a) mapa de la cadena de valor (VSM), b) $5 \mathrm{~s}$ (cinco eses), c) JIT: justo a tiempo, d) Kaizen: mejora continua, e)TQM: calidad total, f) TOC: teoría de las restricciones $\mathrm{g}$ ) reingeniería de procesos, h) manufactura flexible e, i) TPM: mantenimiento productivo total, entre otros. La aplicación de las herramientas del lean manufacturing a través de la práctica, por ejemplo en la aplicación de la metodología de las cinco eses (5 s) con consideraciones de mantenimiento y criticidad de equipos, puede resolver problemas de orden y limpieza de la planta así como el control de maquinarias y equipos que, por no llevar una adecuada trazabilidad en sus actividades, no permita darle estabilidad al proceso y proyectar a la organización a esquemas de planeación y crecimiento.

El trabajo en mantenimiento es la tarea que le permite a cualquier organización sostener en el tiempo y estirar de manera positiva la funcionalidad de sus equipos de modo que estén disponibles para operar en las condiciones para los cuales fueron diseñados. Estas tareas deben, y se recomienda, estar alineadas con la misión, visión y objetivos de la organización de manera que estas se cumplan en función del norte de la empresa.

Sus objetivos son, según Muñoz (2008), los siguientes:

- Asegurar que la fracción de tiempo de la planta en operación sea el adecuado (disponibilidad).

- Garantizar que la planta no tenga fallas inesperadas (confiabilidad).

- Garantizar que cuando ocurra una falla esta sea solucionada lo más rápido posible (mantenibilidad).

- Asegurar que la vida útil de los activos sea lo más larga posible.

- Optimizar los recursos que le asigna la empresa.

Ahora bien, más que tareas de mantenimiento, según (Viveros, 2013), la idea es realizar la mejora y hacerlo de un modo práctico y moderado, lo cual implica o abarca todas aquellas actividades técnicas y administrativas cuyo objetivo es alcanzar la máxima disponibilidad al mínimo costo dentro del marco de la prevención de accidentes y el respeto al medio ambiente.

\section{Cinco eses $(\mathbf{5} s)$}

Es una técnica que deriva su nombre de las iniciales de un proceso lógico y por etapas cuyas palabras (en japonés) comienzan todas por la letra ese (s): seiri, seiton, seiso, seiketsu, shitsuke, que significan, 
respectivamente: 1) eliminar lo innecesario, 2) ordenar (cada cosa en su sitio y un sitio para cada cosa), 3) limpiar e inspeccionar, 4) estandarizar (fijar la norma de trabajo para respetarla) y, 5) disciplina (construir autodisciplina y forjar el hábito de comprometerse) (Rajadell, 2010). La técnica se establece y desarrolla a través de 5 pasos, los cuales corresponden a una mecánica de invención japonesa que se refiere a 5 estados que llevan a la disciplina o mantenimiento de condiciones idóneas de trabajo. Las $5 \mathrm{~s}$, como técnica, también implica para su avance asignación de recursos, mejorar la cultura de la empresa y la consideración de aspectos humanos. En la literatura encontramos ejemplos claros en donde notamos que el objetivo o la meta de esta técnica, según Hirano (1996), es la de acrecentar y mantener las condiciones de trabajo de toda la organización, que permitan contribuir a la seguridad de los trabajadores, el clima laboral, la motivación personal y la eficiencia con el fin de mejorar la calidad del producto, la productividad de la empresa y la competitividad en el mercado.

En la actualidad la herramienta de las $5 \mathrm{~s}$ ha llegado a convertirse en una regla muy importante para las empresas, sin importar cuál fuere su fuente de trabajo o mercado, meta 0 enfoque, es decir, es una herramienta que ha dado frutos y por ello puede ser replicada en áreas operativas o administrativas de cualquier organización empresarial. En la Tabla 1 se identifican y caracterizan las etapas del proceso, su concepto y objetivo metodológico de acuerdo con Villaseñor (2007).

\section{Mantenimiento productivo total (TPM)}

Esta estrategia metodológica de trabajo está encaminada a crear un sistema operativo que aumente la eficiencia de todos los equipos que intervienen en el proceso productivo de la empresa para garantizar su correcto funcionamiento, evitando así despilfarro por pérdidas de tiempo al fallar un equipo, que conllevarían al incumplimiento ante los clientes y mayores costos para la empresa. Martínez (2001) y Cuatrescasas y Torrel (2010), sugieren que el TPM no es responsabilidad solo del personal de mantenimiento sino de todos los que interviene en el proceso operativo. Los operarios se deben ocupar de algunas tareas de mantenimiento tales como limpieza, lubricación, ajustes, reaprietes de tornillos y pequeñas reparaciones. Se pretende conseguir con ello que el operario de producción se implique más en el cuidado de la máquina, siendo

Tabla 1. Caracterización de las etapas del proceso $5 \mathrm{~s}$

\begin{tabular}{|c|c|c|c|}
\hline \multicolumn{2}{|c|}{ Denominación } & \multirow{2}{*}{ Concepto } & \multirow{2}{*}{ Objetivo particular } \\
\hline Japonés & Español & & \\
\hline Seiri & Clasificación & $\begin{array}{l}\text { Identificar y separar objetos } \\
\text { innecesarios de aquellos } \\
\text { necesarios }\end{array}$ & $\begin{array}{l}\text { Eliminar del sitio de trabajo objetos } \\
\text { obsoletos y con muy poca utilidad en el } \\
\text { área de trabajo }\end{array}$ \\
\hline Seiton & Orden & Un lugar para cada cosa & Organizar el espacio de modo eficaz \\
\hline Seiso & Limpieza & Suprimir suciedad & $\begin{array}{l}\text { Tener un sitio adecuado y limpio para } \\
\text { laborar }\end{array}$ \\
\hline Seiketsu & Estandarización & $\begin{array}{l}\text { Prevenir aparición de suciedad } \\
\text { v desorden }\end{array}$ & Generar condiciones de estado estable \\
\hline Shitsuke & Disciplina & Mejora continua & Mantener y mejorar las condiciones \\
\hline
\end{tabular}

Fuente: los autores del artículo basados en Villaseñor (2007). 
el objetivo último el de conseguir cero averías. Por lo tanto, se basa en la formación, motivación e implicación del equipo humano, en lugar de la tecnología (Tavares, 2011). La estrategia del TPM presenta un concepto de "eficiencia operacional", obtenida por el producto y rendimiento de tres indicadores: disponibilidad de equipos, rendimiento de los encargados de la operación y el mantenimiento/calidad de los servicios y productos; indicador útil para medir el rendimiento colectivo del sistema e incluso compararse con las otras empresas del sector. En la Figura 1 (de acuerdo a Arata, 2009) se muestra el ciclo de trabajo del mantenimiento.

En el análisis se definen e identifican las variables dependientes e independientes que hacen que el problema de investigación sea claramente manejable a través del cálculo e interpretación de ellas; a saber:

- Variable dependiente (productividad): según Herrera y Gómez (2018), la productividad se conoce como la relación entre el nivel de producción total y la utilización de los recursos pertinentes para alcanzar el nivel de producción, en otras palabras, la razón entre las salidas y las entradas. Medina (2010) plantea la situación como la forma en cómo se utilizan los insumos de producción durante la elaboración de productos y servicios para satisfacer las necesidades de la sociedad y además establece que es un factor estratégico en las organizaciones, ya que los bienes y los servicios no pueden ser competitivos si no se elaboran con estándares altos de productividad. Esta variable es el resultado del producto o servicio que se da mediante la eficiencia y el uso adecuado de los recursos, siendo uno de ellos el cumplimiento del tiempo pactado, y mediante la eficacia se logra la producción que se necesita en un determinado tiempo.

- Variable independiente (mantenimiento productivo total). Dentro del análisis de este aspecto es importante resaltar los conceptos de: a) confiabilidad: es la probabilidad de un óptimo funcionamiento de una máquina o proceso en ciertas condiciones

Figura 1. Ciclo de trabajo del mantenimiento

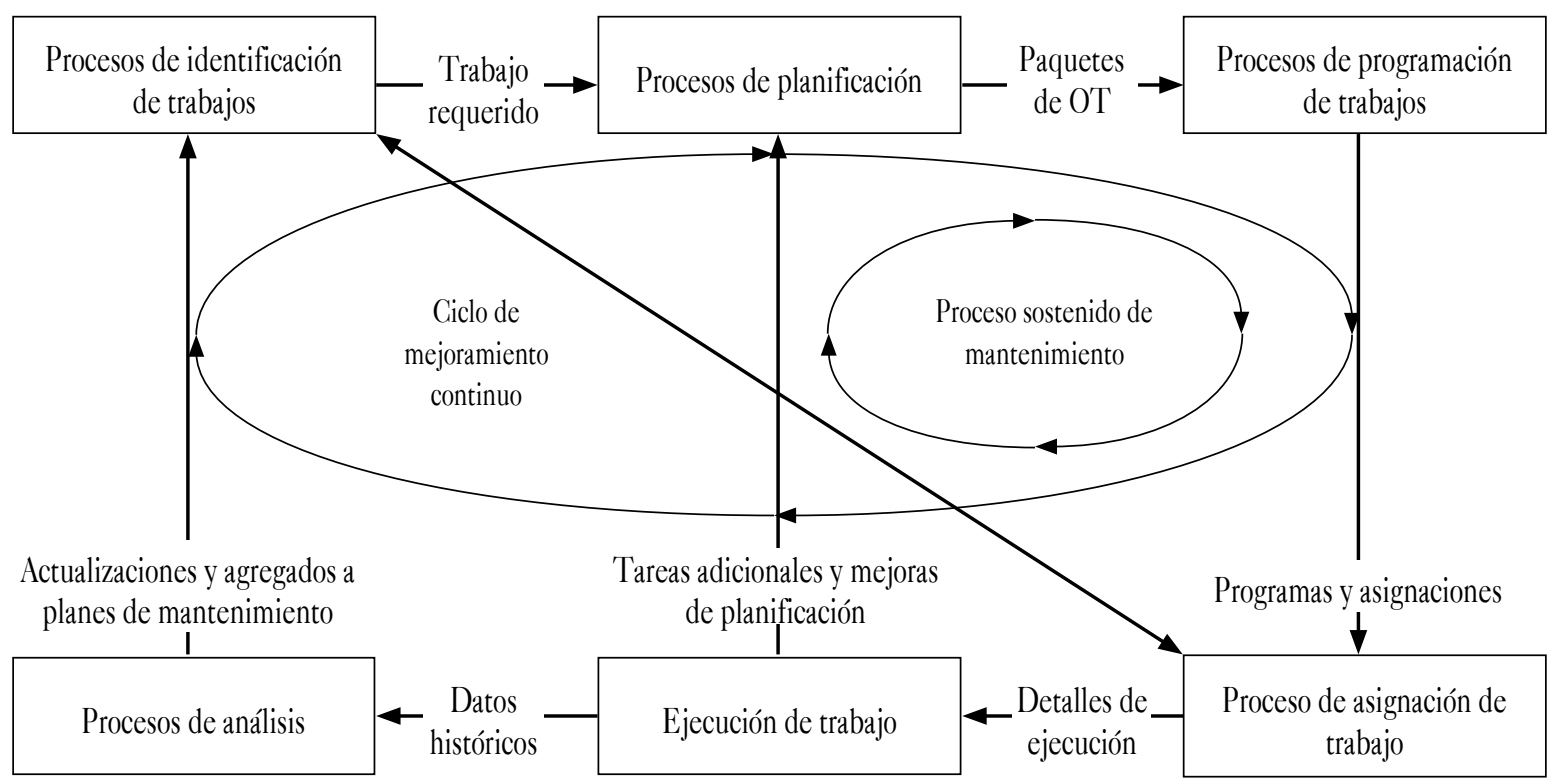

Fuente: tomado de Arata, 2009. 
y durante una etapa determinada, también se puede definir como el tiempo promedio entre fallas (Cossta, 2015) y, b) eficiencia: es la relación que se guarda entre los recursos que se usan y los productos o servicios que se obtienen (Kramis, 1994). Así mismo, y según Allen (2001), este sistema promueve una comunicación efectiva en toda la organización mediante la creación de un lenguaje visual para todo el lugar de trabajo.

\section{ANTECEDENTES}

Cartagena es considerada la primera ciudad industrial del Caribe colombiano, por ser la primera plataforma exportadora de Colombia, por su infraestructura portuaria más eficiente, moderna y segura del país, y por tener cuatro importantes clústeres en su apuesta productiva, entre los cuales está el metalmecánico, entre otros factores, de acuerdo con un estudio realizado por la Universidad de los Andes y la Agencia de Inversiones de Bolívar a finales del 2017 (Universidad de los Andes, 2017). Asílo indican, por lo que el departamento de Bolívar obtuvo un crecimiento del $11.4 \%$, el mayor del país y por encima del promedio nacional, participando con el $8.6 \%$ de la industria manufacturera de Colombia en el PIB. Según datos del Dane, en 2016 movilizó la no despreciable suma de suma de \$ 12.071 millones de dólares generando un ingreso per cápita de $\$ 5.688$ dólares, llevando al departamento a participar del $4.2 \%$ del PIB de Colombia; esto gracias a su industria y a proyectos de gran envergadura como los de la refinería de Cartagena. En la Figura 2 se muestra la actividad económica en el PIB de Bolívar de los sectores en los que se generó empleo en el año 2015 en Cartagena.

Es por lo tanto evidente que en el contexto nacional y local, la actividad metalmecánica además de presentar una oferta laboral fuerte, es importante por su aporte a las diferentes industrias a través del diseño y construcción de partes, estructuras y maquinaria industrial, entre otros, por lo que es pertinente incorporar estrategias y formas de trabajo que potencialicen el desarrollo de la industria y apalanquen el progreso de la

Figura 2. Participación de actividades económicas en el PIB de Bolívar (2015)

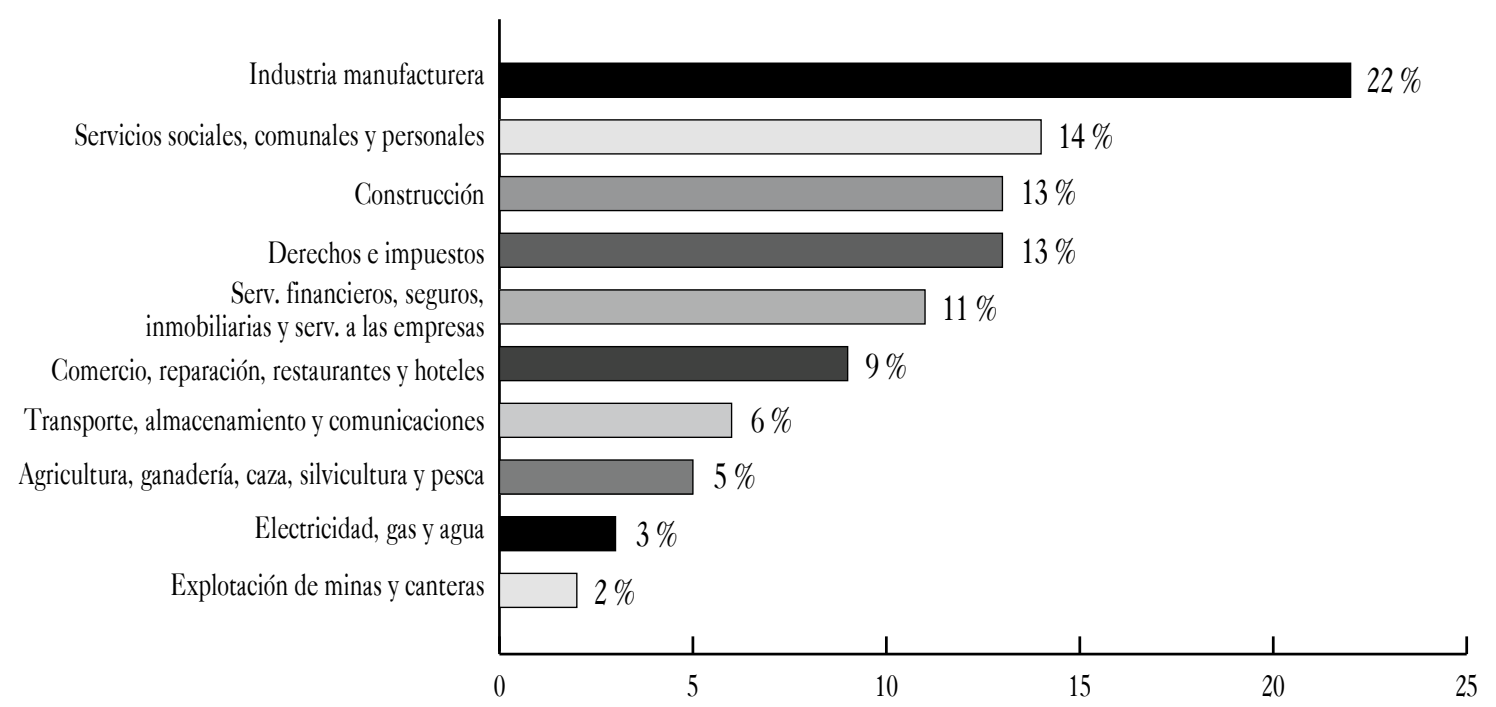

Fuente: Universidad de los Andes. Tomado de https://uniandes.edu.co/sites/default/files/asset/document/Cartagena-2017_0.pdf 
ciudad, teniendo en cuenta que la cadena productiva de la metalmecánica en Cartagena presenta niveles de desarrollo bajos, limitándose a la producción de partes e instalaciones para el sector industrial de Mamonal y fabricación de partes a talleres de la industria automotriz (González y Álvarez, 2012).

En los últimos años se han llevado a cabo varios proyectos para la implementación en la ciudad y en el país de metodologías de apoyo y ayuda a los procesos que impacten en la productividad de las empresas, no obstante, en la mayoría de los casos estos se han limitado principalmente a sectores económicos como el automotor y a otros como el de la construcción. Según Camacol (2015, mayo 20), en empresas como Triada, Urbansa, Arpro, Arrecife y Construmax, se han implementado mejoras a través de la metodología de lean construction o construcción sin pérdidas, logrando eliminar desperdicios en los procesos constructivos y el control y planificación de los proyectos.

\section{METODOLOGÍA}

La implementación del lean manufacturingen Cartagena, Colombia, no es nuevo. En los últimos cinco años y como se mencionó anteriormente, se han elaborado varios estudios para la implantación en la ciudad y en el país de esta metodología, aunque principalmente en sectores como el automotor y el de la construcción, Es por ello que se desarrolló una metodología de aplicación con herramientas lean para una empresa metalmecánica en Cartagena, el cual se realizó en cuatro fases, las cuales se describen a continuación:

- Fase 0. Autodiagnóstico de la empresa y análisis externo: este se realizó utilizando el modelo de red de tecnología INTI, lista de chequeo y análisis documental.

- Fase 1. Diagnóstico inicial: revisión de herramientas por observación directa y uso de herramienta de diagnóstico, entrevistas al personal y recorrido por las instalaciones.

- Fase 2. Aplicación y análisis de las técnicas escogidas de común acuerdo con la dirección: 5 s y TPM.

- Fase 3. Evaluación de la implementación de las herramientas mediante el análisis de resultados y el contraste con datos de simulación del estado estadístico asociado a la distribución de probabilidad Weibull.

En primera instancia, se realizó un autodiagnóstico general (Fase 0) sobre las diferentes áreas de gestión de la empresa utilizando el modelo de la red de Tecnologías de Gestión del Institutito Nacional de Tecnología Industrial INTI de Argentina, en octubre de 2017, a través de un enlace web en https://www.inti.gob.ar/tecnologiasdegestion/pdf/AutodiagnosticoEmpresarial. xls. Este autodiagnóstico ha venido siendo aplicado a diferentes empresas del sector metalmecánico en Cartagena, dado que este instrumento es público y puede ser utilizado por cualquier empresa. Fue el insumo inicial y que junto con otro instrumento general o lista de chequeo con diversas herramientas lean, ayudaron a establecer los elementos de la Fase 0.

Entre las estrategias lean identificadas y aplicables a la empresa, teniendo en cuenta los criterios de esta para priorizar aquellas con mayor impacto en el corto plazo utilizando recursos mínimos con el fin de motivar y estimular al personal para continuar con el proceso de cambio y mejora a todas las diferentes áreas de la organización, se identifican 5 s y TPM como las más relevantes. Este autodiagnóstico y lista de chequeo también permite un sistema de ayudas para organizar y controlar el plan de trabajo, contribuir a asegurar calidad y proporcionar apoyo en la construcción de estándares de productividad para la empresa.

Una vez realizada esta fase de autodiagnóstico, se procedió a la Fase 1, en la cual se aplicó una encuesta a 
todos los operarios de producción, área en la cual se centrará la propuesta de mejora de esta investigación. También se utilizó una hoja de flujo del proceso para describir los procesos de fabricación de un producto representativo de la producción en la empresa. Con la aplicación de la encuesta y la hoja de flujo de proceso, se fueron tomando observaciones de la forma como estaban organizados los puestos, aseo, orden, clasificación de las herramientas de trabajo y demoras, entre otras, información importante para detectar aspectos por mejorar y completar el diagnóstico.

Para el desarrollo, se hace necesaria la aplicación de las herramientas como la lista de chequeo y observación directa, realizando las visitas periódicas de campo a la empresa, las entrevistas al personal y encuestas realizadas directamente con los usuarios, con el objeto de aplicar las herramientas lean escogidas de acuerdo con la situación actual del caso objeto de estudio. Luego de esto y para llevar a cabo la Fase 2, se trabajó bajo el apoyo metodológico ofrecido por la metodología de las 5 s. Posteriormente, se analizan las condiciones operativas y de mantenimiento de modo que se pueda levantar una estrategia TPM. Finalmente, se evalúa el proceso utilizando indicadores de rendimiento y gestión, lo que corresponde a la Fase 3.

\section{RESULTADOS Y DISCUSIÓN}

A continuación, se presentan los resultados de la aplicación de la metodología.

\section{Bases para el diagnóstico inicial}

En el caso de estudio en la empresa escogida, después de aplicado el instrumento y la lista de chequeo en algunas áreas, se encontraron fortalezas, pero el resultado no arrojó una buena calificación (2.4 de un total de 5.0 puntos en una escala ordinal), ya que se detectaron algunas deficiencias en infraestructura, inventario, materiales y proveedores, maquinaria, procesos, calidad y manejo de residuos, como se muestra en la Figura 3 del autodiagnóstico para el área de producción. Adicionalmente, una de las variables más críticas es el manejo y disposición de residuos, con la calificación más baja correspondiente a 1.6 sobre 5 puntos, lo que indica que en el $32 \%$ de las ocasiones en que se lleva a cabo el proceso productivo, los restos como grasa, gasolina, material mecanizado, agua contaminada y otros aditivos necesarios para diluir la grasa, no tienen la disposición adecuada, ocasionando que los lugares de trabajo y las máquinas con que se trabaja presenten condiciones de orden y aseo bastante desagradables, lo que podría propiciar además de accidentes de trabajo, tiempos perdidos y daños a los equipos afectando todo esto la productividad.

Así mismo, los lugares de ubicación de los materiales en proceso (partes de máquinas y equipos para reparación) como también los insumos usados en las reparaciones, no tienen un control en los inventarios, impactando de esta manera en la infraestructura, ya que el $46 \%$ de las veces (según la calificación obtenida en el diagnóstico que se aprecia en la Figura 4 sobre el puntaje del área de producción de la empresa), esta área es usada de modo inadecuado, obstaculizando el flujo de materiales y alterando visualmente el ambiente de trabajo, no solo por ubicar equipos y materiales en lugares no acondicionados sino también afectando pisos, paredes y otras máquinas a las que se les da uso indebido.

En la identificación de los aspectos e impactos en el área operativa antes descritos, también se evidenció que, por ejemplo, hay contraflujos y las distancias son apreciables para los productos en inventario (terminados 0 en proceso). No existen lugares señalizados 0 preestablecidos o simplemente los usados son espacios inadecuados. Así mismo, es fácil ver que no existe una 
Fiǵura 3. Autodiagnóstico del área de producción

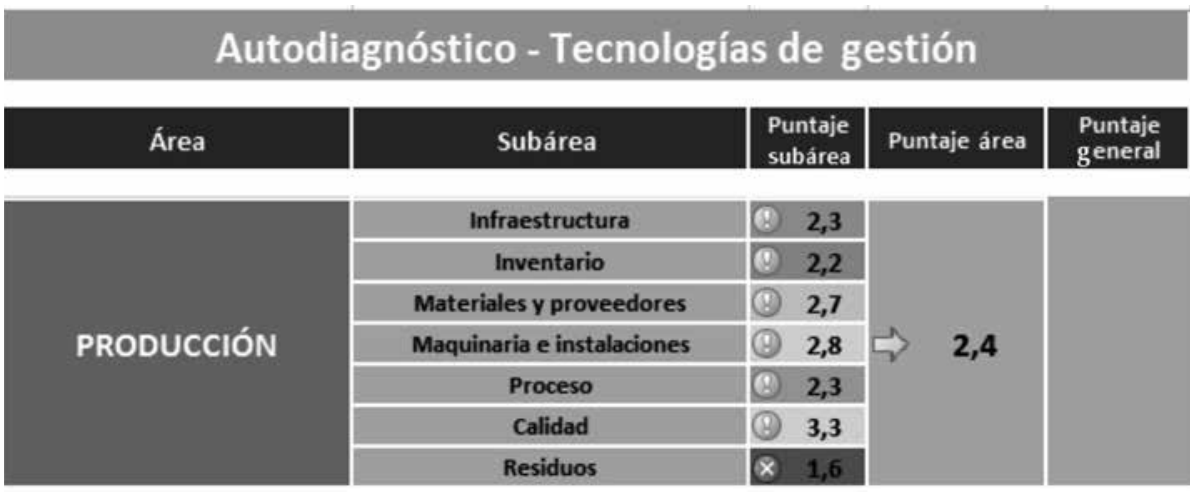

Fuente: los autores del artículo basados en información de la empresa.

política clara del mantenimiento, por lo que el $47 \%$ de los eventos por fallos son imprevistos y por tanto las tareas ejecutadas para la disponibilidad de los equipos es de tipo correctivo; no se analizan las causas y por ello el $52 \%$ de las fallas son recurrentes o repetitivas, eso sin tener en cuenta que no se llevan registros de fallos 0 las causas asociados a las pérdidas de funcionalidad de los equipos. No hay un responsable de la vigilancia tecnológica de modo formal y la información se obtiene solo a través de contactos con proveedores.

Figura 4. Puntaje del área de producción de la empresa

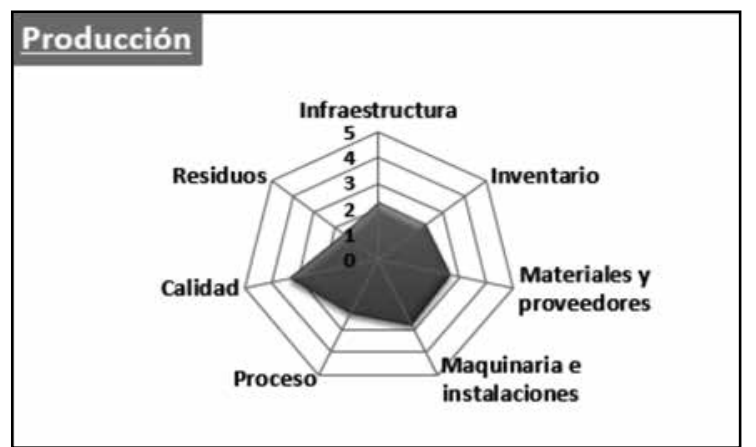

Fuente: los autores del artículo basados en información de la empresa.

Teniendo en cuenta que la problemática central del proceso operativo es la falta de técnicas de organización, manejo de residuos, infraestructura, manejo de inventario, materiales y proveedores, la no existencia de planes de mantenimiento a los equipos e instalaciones, efectividad de los procesos y control sobre la calidad operacional; es determinante conocer las causas que hacen que este problema persista en la empresa. Es por ello que, con una adecuada gestión de mantenimiento, es fundamental la conveniente operación de los equipos, que debe ser considerada desde las etapas tempranas tanto en el diseño o compra de estos, lo que se denominada gestión temprana, y el análisis en materiales, lubricantes y nuevas técnicas de análisis en los equipos para optimizar en forma constante los recursos disponibles.

De igual manera, en el análisis se dedujo que existen dos causas directas: la primera está relacionada con la falta de organización y manejo de residuos en el modo en que se ejecutan las tareas debido a la falta de estructuración del layout (en español: diseño, plan, disposición) de la planta y la actitud de los operadores en su rutina de trabajo. La segunda causa directa está asociada con el inadecuado uso operativo de los equipos, restringiendo su operatividad y afectando e impactando negativamente la infraestructura y vida útil de los mismos.

De lo anterior se concluye que se requiere iniciar una estrategia de mejora a partir de la aplicación de $5 \mathrm{~s}$ y TPM para resguardar los activos de la empresa y propiciar la creación de nuevas políticas que propendan por la mejora de los diferentes aspectos estudiados y 
un cambio en la actitud de todos los trabajadores en la empresa caso de estudio en la investigación.

\section{Aplicando 5 s y TPM}

Para la implementación de la metodología $5 \mathrm{~s}$, se comienza con una capacitación en esta temática, posteriormente se escoge el área piloto para el establecimiento que fue el espacio destinado para el lavado de las piezas; seguidamente se toma evidencia fotográfica del estado actual del área de lavado y se levanta el plano de la planta; luego se realiza un listado de los objetos presentes en el sitio de trabajo y se procede a clasificar los objetos por medio de tarjetas rojas; con esto se logran eliminar y reubicar objetos que no son necesarios en este espacio y de esta forma se obtiene un lugar más organizado, limpio y despejado para la circulación del operario y las piezas a trabajar.

Es importante aclarar que este proceso es de difícil inicio, romper viejos paradigmas mentales en los empleados es algo complicado, pero del mismo modo, cobra vital importancia una vez formalizado por su impacto visual que hace el contraste en el antes y el después de aplicar la técnica de las $5 \mathrm{~s}$ en la motivación de los operarios. Adicionalmente, el apoyo gerencial fue vital para que las tareas de orden, clasificación y limpieza empezaran a dar buenos resultados. De la mano de este proceso se hace necesario implementar medidas que, en la parte de mantenimiento, mitiguen los impactos negativos sobre las máquinas y en el proceso en la prestación de los servicios.

La empresa inicia el proceso de implementación de las $5 \mathrm{~s}$ a partir de febrero del 2018, logrando implementar hasta la $3 \mathrm{~s}$ en un plazo de 4 meses. El área escogida como piloto es la correspondiente a la operación de lavado. En este lugar se lograron listar los objetos, de los cuales un $55 \%$ (peso de $37.1 \mathrm{~kg}$ ) fue eliminado, liberándose un espacio de $2.89 \mathrm{~m}^{2}$ y reduciendose el recorrido del trabajador en $9.65 \mathrm{~m}$.
Para el caso de los trabajos de mantenimiento, de acuerdo a criterios TPM, los reportes de los estudios que se iniciaron en el área de producción (operaciones) nos muestran que aquellas máquinas con mayores dificultades son: 1) Rectificadora de asientos CNC: el pistón hidráulico no sujeta bien la pieza llamada piloto por ausencia de tornillo hidráulico, la máquina opera con pistón hidráulico reconstruido por el personal de mantenimiento desde hace dos años y el cambio de mangueras de la máquina se realiza de forma frecuente por daños en las mismas (cada 2 meses aprox.), entre los antecedentes más importantes. 2) Rectificadora de cigüeñal: la falta de empaque de válvula doxa genera el goteo de aceite que llega a contaminar el agua y conlleva a fallas en el cabezal por roturas, los rodamientos deben estar siempre lubricados para evitar desgaste en las piezas de las máquinas. 3) Torno $\mathrm{N}^{\circ} 2$ ZMM VRATZA 582: el interruptor de encendido no funcionaba por lo que la máquina se encendía de forma directa al conectarla. Se le realiza el arreglo al interruptor interno lo que permite el encendido normal de la máquina. La parte magnética del contador de la máquina está fallando. 4) Pulidor de cilindro CNC: Los vanadores se queman debido a la fluctuación de energía. Esta circunstancia hace que el equipo presente fallos intermitentes pero ininterrumpidos desde hace dos años aproximadamente.

Una vez identificados los equipos de mayor mantenimiento en el área operativa y que por ello se convierten en el primer escalón de atención para el proceso productivo, se inicia un análisis que permita conocer los modos de fallos y sus consecuencias de forma que los trabajos de mantenimiento apunten a la mitigación de los mismo de manera inmediata y se programen las tareas y mecanismos de planeación de las tareas y rutinas para prevenir la reaparición de los mismos y de esa forma tener un mejor control operativo. 
Martha Sofía Carrillo Landazábal, Carmen Giarma Alvis Ruiz, Yaniris Yaneth Mendoza Álvarez, Harold Enrique Cohen Padilla

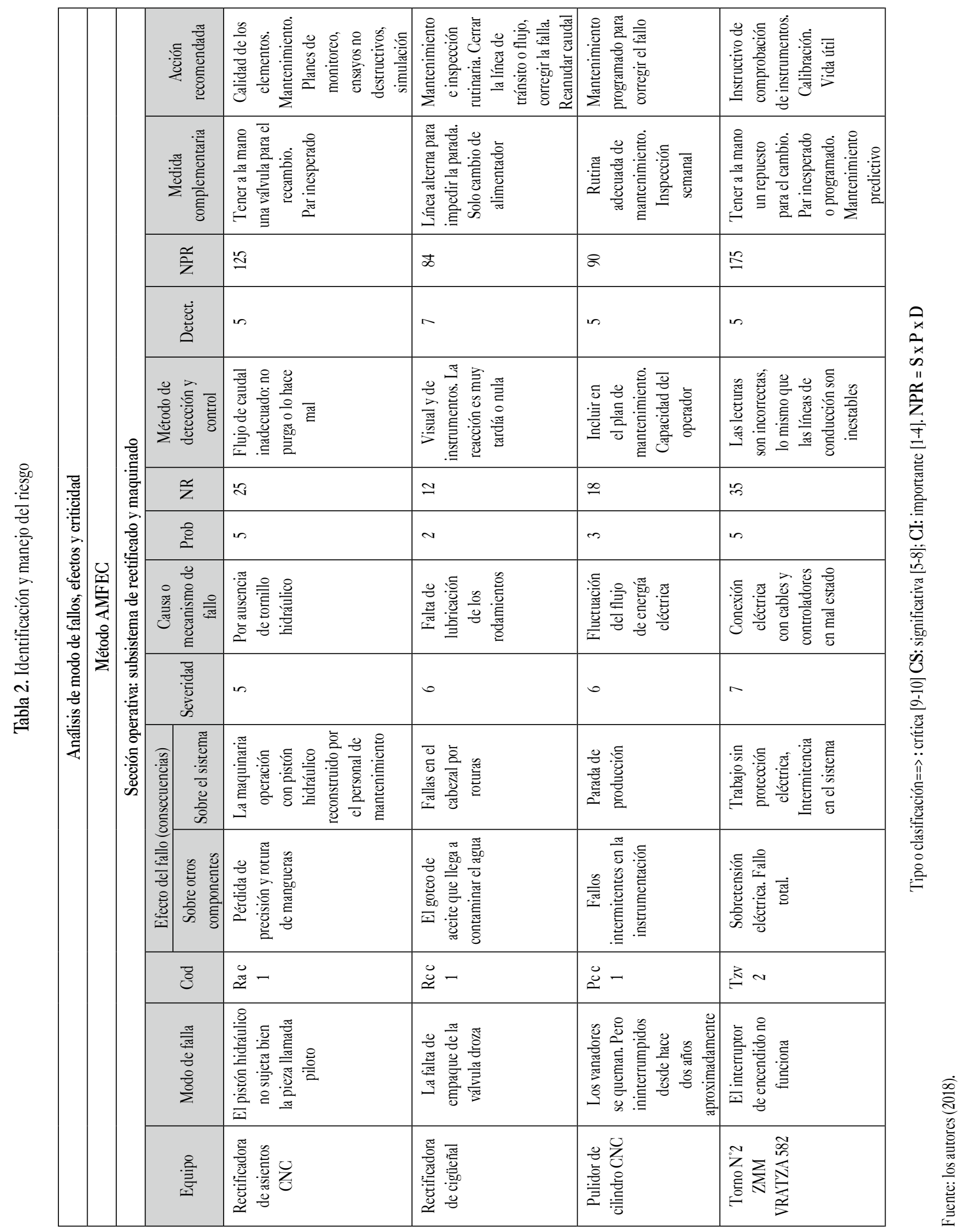


Luego del análisis se detecta que el NPR o número de probabilidad del riesgo y su impacto más crítico se encuentra en el primero y cuarto equipos estudiados, es decir, la rectificadora de asientos $\mathrm{CNC}$ y el torno $\mathrm{N}^{\circ} 2$ ZMM VRATZA 582, equipos sobre los cuales se recomienda hacer un énfasis en las tareas de mantenimiento.

\section{Evaluación y análisis en contraste con una simulación}

El ingeniero Carlos Sierra Fernández, Ph. D., explica, dentro de las funciones de la teoría general del mantenimiento y de la fiabilidad de la cátedra de Técnicas de Mantenimiento en Instalaciones Mineras que imparte en la Universidad de Cantabria, que "en teoría de la probabilidad y estadística, la distribución de Weibull es una distribución de probabilidad continua y multiparamétrica. El campo de la fiabilidad y el mantenimiento es la más utilizada y una de las que aportan mayor precisión".

En esta fase se analizan datos históricos de fallas y de reparación, asignando distribuciones de probabilidad que representan las características de falla o de reparación de un modo determinado. A partir de los datos encontrados de los últimos fallos registrados, se hace una estimación estadística simulada del comportamiento de dichos fallos intentando predecir por tendencias de aproximación de los modelos preestablecidos, tal como se muestra en la Tabla 3 y el cálculo de $\mathrm{R}(\mathrm{t})$ en la Figura 5. Es decir, con ayuda del software y una correlación de variables, atendiendo a la simulación de Montecarlo, se hace una extrapolación de condiciones de manera que se obtienen valores aleatorios que intenten representar estadísticamente el comportamiento de los últimos fallos registrados y evidenciados en los reportes entregados por los encargados del mantenimiento en la empresa estudiada.

Los datos simulados representan el modelo (cosa que se evidencia en la tendencia de la gráfica), por lo que podemos asumir que la simulación se aproxima a lo real, ya que hay bastante similitud de los datos reales con los simulados ya que la dispersión es poca (Figura 6). Lo ideal es que la dispersión de los datos sea más estrecha que la dispersión de especificación y que todos los datos estén dentro de los límites de especificación. Los datos que están fuera de los límites de especificación representan elementos no conformes. En esta figura de la distribución Weibull (última representación), se

Tabla 3. Tiempos de fallos (MTTR) simulados en Weibull ++6

\begin{tabular}{|r|r|r|r|r|r|r|r|r|r|}
\hline $6,54 \mathrm{E}-03$ & 2,51027581 & 8,26025868 & 16,8093931 & 28,4525989 & 52,7673254 & 119,047686 & 191,504605 & 287,226897 & 463,282406 \\
\hline 0,28158417 & 2,7274968 & 8,63325672 & 16,9294633 & 32,8727314 & 54,2813155 & 120,193962 & 196,236405 & 287,818034 & 576,838263 \\
\hline 0,51705871 & 2,97519542 & 8,68155069 & 16,9597548 & 33,185482 & 54,2823797 & 138,088525 & 205,056227 & 297,818034 & 612,635308 \\
\hline 0,61891635 & 3,73044492 & 9,5051323 & 18,4643917 & 36,1273065 & 61,7576044 & 141,470085 & 209,0203 & 322,691079 & 722,721555 \\
\hline 0,63960783 & 4,91327752 & 10,6140247 & 18,543423 & 37,0956071 & 69,850806 & 150,774672 & 214,419043 & 323,988986 & 806,427335 \\
\hline 1,18392641 & 6,16501292 & 10,8516689 & 18,7129705 & 41,5224408 & 82,6801671 & 158,852858 & 219,12587 & 337,953395 & 984,183394 \\
\hline 1,29079868 & 6,95603982 & 11,2563069 & 22,6044761 & 47,2578834 & 85,054046 & 175,77293 & 229,835597 & 370,11112 & 1075,24638 \\
\hline 1,81242679 & 7,30334255 & 12,7746771 & 25,3042512 & 48,496275 & 92,0480641 & 177,684833 & 274,999974 & 410,325658 & 1076,67344 \\
\hline 1,97126781 & 7,69904287 & 13,7698388 & 27,235951 & 50,1209392 & 92,4986069 & 179,310406 & 279,550493 & 437,975029 & 1756,9221 \\
\hline 2,28366587 & 8,14027465 & 14,5042897 & 28,1159287 & 50,8263532 & 97,1901909 & 187,66875 & 284,436795 & 442,585674 & 4021,14956 \\
\hline
\end{tabular}

Fuente: resultados del software Weibull ++6 con datos de la empresa. 
Figura 5. Cálculo de R(t) Fiabilidad - Distribución de probabilidad Weibull

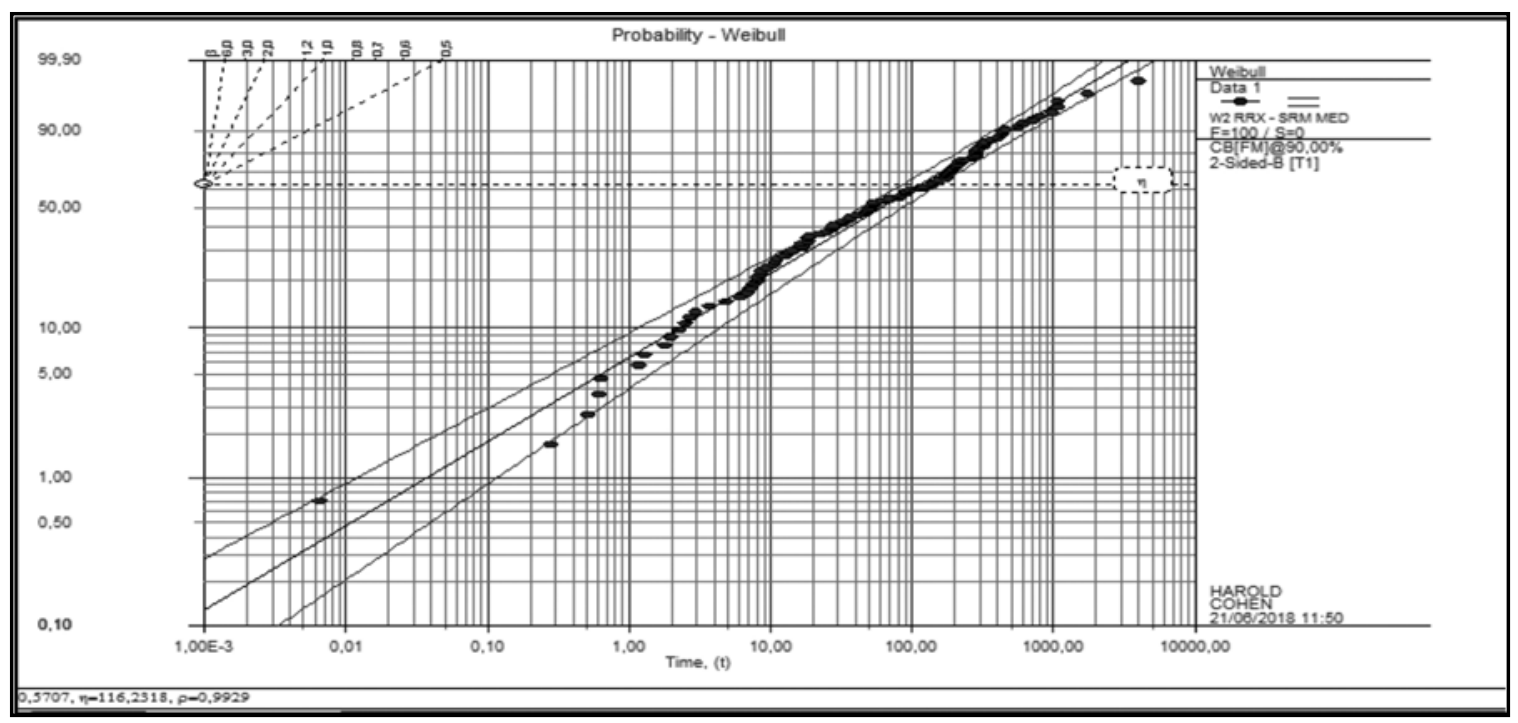

Fuente: resultados del software Weibull ++6 con datos cargados por los autores.

puede observar que la dispersión del proceso es mayor que la dispersión de especificación, lo que sugiere una capacidad deficiente. Aunque gran parte de los datos se encuentran dentro de los límites de especificación, hay muchos elementos no conformes por debajo del límite de especificación inferior, lo que hace que la tendencia de los datos se sitúe al lado izquierdo.

De los resultados obtenidos del software podemos verificar que cuando el parámetro beta (B) es $>1$, se dice que la tasa de fallo se incrementa con la edad de forma continua, lo que indica que los desgastes empiezan en el momento en que el mecanismo se pone en servicio. Para el caso de estudio se presenta esta situación, por lo que se infiere que las piezas de los equipos de la empresa muestran fallos debido a estas circunstancias, que se evidencia en la hoja de vida de estas (Figura 6).

En la Figura 6 podemos validar que hay un $90 \%$ de posibilidades de que el tiempo de fallos sea menor a 4021 minutos, que es el dato máximo posible (según la simulación), dado que el rango medio para este último punto es de 0.90 . Usualmente, la vida característica $\prod$ es el valor del dato (en este caso: MTTR) que corresponde al $57 \%$ aprox. del valor del rango medio de la línea recta.

\section{CONCLUSIONES}

- Un sitio aseado no es aquel que debe limpiarse mucho sino todo lo contrario, y eso es algo que han entendido los operadores, por tanto, el orden, la limpieza y el aseo es una actividad que, sin ser fácil, se ha convertido en la herramienta de motivación en la empresa. Razón por la cual el aspecto visual ha ayudado a la operación y a tareas de mantenimiento en pro, incluso, de la producción. Es por eso por lo que la empresa ha iniciado el proceso de implementación de las $5 \mathrm{~s}$ en los últimos 4 meses, logrando en las primeras $3 \mathrm{~s}$, para el área piloto escogida (lavado), un total de material eliminado de $37,1 \mathrm{~kg}$; espacio despejado correspondiente a un $22 \%$ del total del área intervenida con la metodología; sin embargo, para mantener estas 
Figura 6. Gráficas Weibull: tasa de fallos - Fiabilidad acumulada Fiabilidad - Distribución de probabilidad Weibull

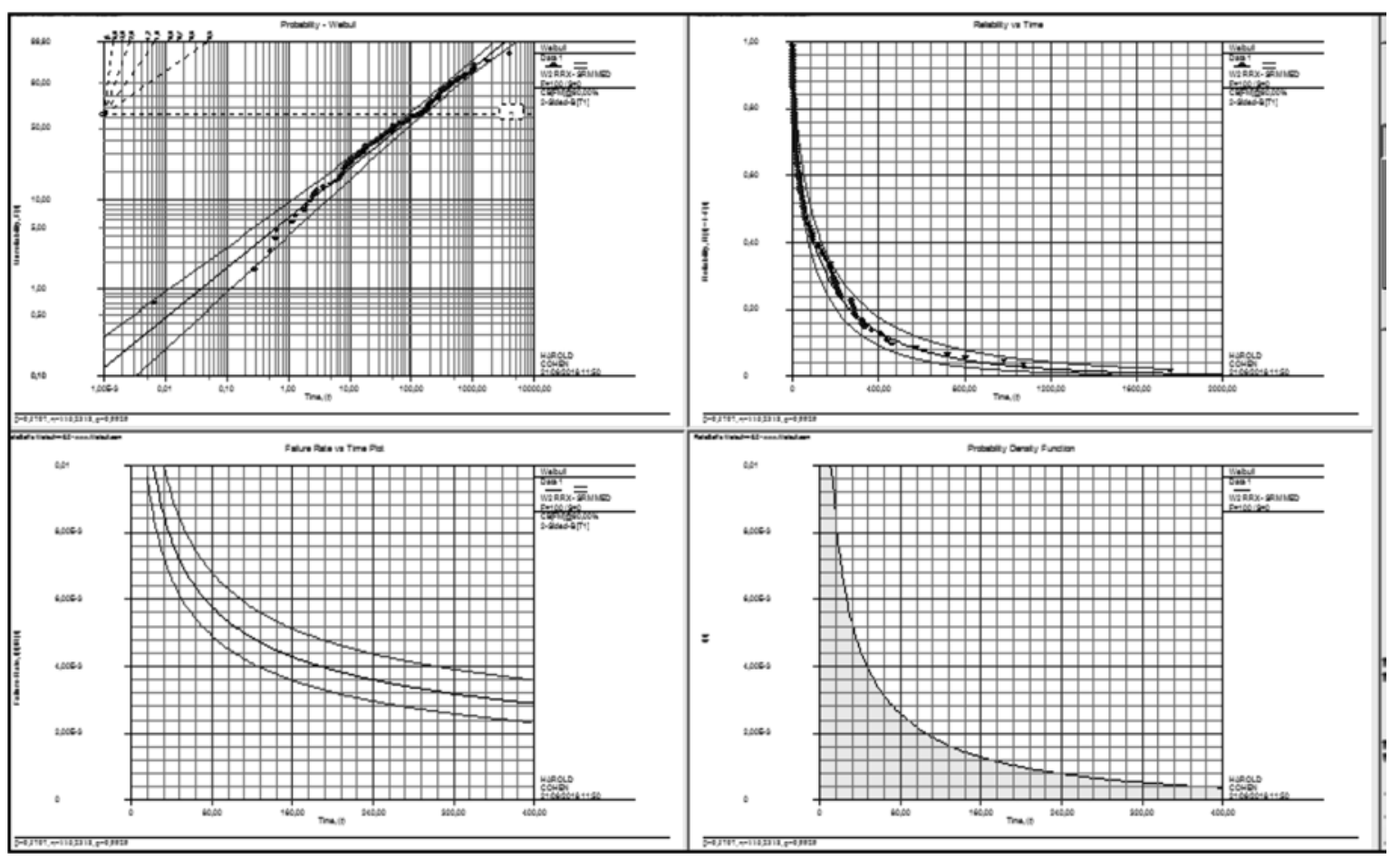

Fuente: resultados del software para Weibull con datos cargados por los autores.

condiciones se hace necesario buscar otras alternativas de mejora y culminar la implementación de las dos "s" restantes.

- Las probabilidades de fallo están muy cerca del 50 $\%$, lo que infiere que la mitad de las veces un equipo es susceptible de fallar. En ese orden de ideas se proponen rutinas de inspección de equipos que mitiguen los mantenimientos correctivos y así reducir de $47 \%$ (valor actual) a lo menos un $20 \%$ las probabilidades de fallo (en una primera etapa: próximo mes) e incluso a un $10 \%$ luego de una revisión y ajuste un par de meses luego de iniciado el proceso lean.

- Los datos históricos y el análisis de modos de fallos demostraron que los fallos más recurrentes están asociados a la manera no adecuada en que se hacen las operaciones productivas y las rutinas de alistamiento de las máquinas, por lo que, si se adaptan mejores prácticas operativas de cuidado y mantenimiento de las máquinas cuando se realizan las actividades, se reducirá ostensiblemente la criticidad y la frecuencia de los fallos. Por lo tanto, se recomienda un plan de capacitación y actualización del plan de mantenimiento para operadores y equipo de supervisión de tareas de la empresa.

- Programar las tareas de mantenimiento junto con aquellas que ameriten paradas de equipo, de modo que se optimicen no solo los tiempos sino también los recursos. Así, se solicita que la gestión administrativa, tanto de compras como humana, coordine tareas de tal manera que el TPM funcione de forma adecuada. 
Martha Sofía Carrillo Landazábal, Carmen Giarma Alvis Ruiz, Yaniris Yaneth Mendoza Álvarez, Harold Enrique Cohen Padilla

\section{REFERENCIAS}

Allen J., R. C. (2001). Lean Manufacturing: A plant floor guide. Society of Manufacturing Engineers, 276 p.

Arata. A. (2009). Ingeniería y gestión de la confiabilidad operacional en plantas industriales. Santiago de Chile, Chile: RIL Editores.

Camacol. (2015, mayo 20). Fundador de lean construction entrega reconocimiento a empresas colombianas que implementaron el modelo. Recuperado de https://ww2.camacolcundinamarca.co/382-reconocimiento-lean-construction.html, F

Cossta, G. G. (2015). Elaboración de un plan de mejora para el mantenimiento preventivo en los sistemas de aire acondicionado de la red de telefónica del Perú, Zonal Norte, basado en la metodología Ishikawa-Pareto (tesis de pregrado). Universidad Privada Antenor Orrego, Trujillo, Perú.

Cuatrescasas, L., y Torrel F. (2010). TPM en un entorno lean management: estrategia competitiva. Madrid, España: Profit Editorial.

Gónzalez, J., y Álvarez, A. (2012). La gestión empresarial de las micro, pequeñas y medianas empresas del subsector metalmecánico de Cartagena en el período 2004-2010. Revista Saber, Ciencia y Libertad, 111-118.

Herrera, T. F., y Gómez, J. (2018). La productividad y sus factores: incidencia en el mejoramiento organizacional. Dimensión empresarial, 16(1), 47-60.

Hirano, H. (1996). 5 s for operators: 5 pillars of the visual workplace. New York: Productivity Press.

Holweg, M. ( 2007). The genealogy of lean production. Journal of Operations Management, 25(2), 420-437.
Kramis, J. (1994). Sistemas y procedimientos administrativos. Mexico D. F., México: Universidad Iberoamericana.

Martínez, A. P. (2001). Lean indicators and manufacturing strategies. Lean indicators an International Journal of Operations \& Production Management, 21(11), 1433-1452.

Muñoz, M. (2008). Mantenimiento industrial. Madrid, España: Universidad Carlos III de Madrid, Área de Ingeniería Mecánica.

Rajadell, M. (2010). Lean manufacturing, la evidencia de una necesidad. Madrid, España: Editorial Díaz de Santos.

Shah, R., y Ward, P. T. (2007). Defining and developing measures of lean production. Journal of operations management, 25(4), 785-805.

Stone, K. F. (2012). Decades of lean: A systematic literature review. International Journal of Lean Six Sigma, 3(2),112-132.

Tavares, L. (2011). Gestión de activos para el mantenimiento. Mantenimiento mundial. Recuperado de http://www.conexionmantenimiento.com/articulos/gestion.pdf.

Viveros, P. S. (2013). Propuesta de un modelo de gestión de mantenimiento y sus principales herramientas de apoyo. Ingeniare. Revista Chilena de Ingeniería, 21(1), 125-138.

Villaseñor, A. (2007). Manual de lean manufacturing. México, D. F., México: Editorial Limusa S. A. Grupo Noriega Editores. 